\title{
Sistema photogate de seis canais analógicos para laboratórios didáticos de física
}

\author{
Photogate system with six analogic channels suitable for physics learning laboratories \\ João T. de Carvalho Neto*1, Fernando R. Apolinário ${ }^{1}$, Aline de A. Soares ${ }^{1}$ \\ ${ }^{1}$ Departamento de Ciências da Natureza, Matemática e Educação, Universidade Federal de São Carlos, São Paulo, Brasil.
}

Recebido em 22 de Maio de 2017. Aceito em 7 de Julho de 2017

\begin{abstract}
Descrevemos e disponibilizamos um sistema de chaves óticas (photogates) completo (hardware + software) para uso em laboratórios didáticos de física. Conjugamos diferentes abordagens propostas na literatura em um conjunto que oferece boa resolução temporal $(\approx 1 \mathrm{~ms})$, alto número de chaves óticas independentes (até seis canais), alta sensibilidade (conversão analógica-digital de até 10 bits), baixo custo (hardware e software livres ou de uso gratuito) e simplicidade de montagem. O sistema foi testado em três experimentos clássicos de ensino de física: (i) plano inclinado, (ii) viscosímetro e (iii) pêndulo simples. Acreditamos que as ferramentas aqui descritas ajudem a contribuir na estruturação e autonomia de laboratórios de ensino ao propiciar o desenvolvimento de projetos robustos com qualidade e versatilidade iguais ou superiores às opções comerciais.
\end{abstract}

Palavras-chave: portas óticas, arduino, ferramentas digitais, laboratórios didáticos.

The development and deployment of a complete photogate system (hardware + software) to be used in physics learning laboratories is described. Different approaches found in the literature are conjugated in a configuration that offers good time resolution $(\approx 1 \mathrm{~ms})$, high number of independent optical gates (up to six channels), high sensibility (digital-analog conversion up to 10 bits), low cost (open or free hardware and software) and easy assembly. The system was probed for three classic physics learning experiments: (i) inclined plane, (ii) viscosimeter and (iii) simple pendulum. We believe that the described tools can help to improve the learning physics laboratories autonomy and infrastructure, engaging stronger project designs with quality and versatility as good as the commercial options. Keywords: photogates, arduino, digital tools, learning laboratories.

\section{Introdução}

O desenvolvimento de atividades experimentais é parte central das disciplinas pertencentes às ciências naturais [1]. Entretanto, a estruturação e manutenção de um laboratório didático com condições mínimas para o desenvolvimento de experimentos com boa qualidade pode encontrar barreiras nos recursos humanos e financeiros. Do ponto de vista financeiro, não são muitas as opções no mercado nacional de equipamentos didáticos para o ensino de física, o que contribui para o seu encarecimento e baixa diversidade de opções e configurações. Ao mesmo tempo, vem se tornando cada vez mais acessível o desenvolvimento de instrumentação de qualidade, que pode ser voltada ou adaptada para o ensino de ciências, através dos projetos livres e cooperativos voltados à filosofia do it yourself [2]. Um número cada vez maior de plataformas de divulgação e distribuição de projetos encontra-se disponível pela internet. Enquanto a maior parte dessas iniciativas é voltada ao público leigo ou hobista e carece de uma fundamentação técnica ou científica mais sólida, existem propostas mais sofisticadas com aplicações relevantes em ciência e tecnologia 3 .

*Endereço de correspondência: jocoteles@gmail.com
Essas experiências mostram ser possível aproveitar as ferramentas digitais disponíveis para o desenvolvimento e compartilhamento de projetos de instrumentação em ensino de ciências comprometidos com a qualidade e versatilidade necessárias para subsidiar os processos de ensino-aprendizagem nas disciplinas experimentais, sejam da educação básica ou superior. Com relação às restrições de pessoal técnico capacitado, os projetos abertos e colaborativos contribuem para aumentar a acessibilidade e viabilidade do desenvolvimento de instrumentação de apoio às atividades experimentais através do compartilhamento de experiências e recursos audio-visuais, além da documentação técnica.

As chaves óticas ou photogates são dispositivos bastante úteis nos laboratórios de ensino de física pois auxiliam na aferição de intervalos de tempo em vários experimentos voltados aos conteúdos de cinemática, dinâmica, fluidos, entre outros. Várias são as propostas de dispositivos de chaves óticas para o ensino de física, em que se vê claramente a evolução das configurações dos projetos em função das ferramentas e tecnologias disponíveis [4 9]. Baseando-se em nossa revisão da literatura, identificamos quatro critérios sob os quais podemos avaliar os trabalhos propostos: (i) custo financeiro, (ii) facilidade de confecção, 
(iii) funcionalidade e (iv) escopo de aplicação. Por motivos óbvios, a grande maioria enquadra-se na categoria de baixo custo, em que, dependendo da época de publicação do trabalho, tecnologias mais sofisticadas (principalmente dispositivos eletrônicos e opto-eletrônicos) estão disponíveis a custos mais acessíveis. Em muitos casos, existe um compromisso entre o critério de facilidade de confecção e os critérios de funcionalidade e escopo de aplicação: projetos mais simples e de fácil montagem tendem a ser mais restritos em suas funcionalidades, restringindo também sua aplicabilidade a diferentes experimentos e níveis de ensino.

Atualmente, entre as propostas mais simples e de baixo custo estão aquelas que utilizam fototransistores conectados à entrada de microfone de computadores 4, 10. Nesses projetos, a apresentação e manipulação dos dados das chaves óticas é feita usando-se softwares de análise de áudio ou softwares dedicados para esse fim. Uma outra abordagem é a confecção de hardware autônomo - sem necessidade do apoio de um computador - para registrar os dados medidos pelos sensores óticos, como circuitos com contadores e mostradores digitais e cronômetros digitais 6 6 , 8 , 9. Com relação aos sensores de posição, existem abordagens alternativas às chaves óticas que incluem sensores magnéticos e sonoros 11-13.

Nos últimos anos, uma peça de hardware que tem popularizado o desenvolvimento de vários projetos eletrônicos, inclusive na área de ensino, é a plataforma Arduino [3]. Com ela é possível ler diversos sensores analógicos e digitais de forma autônoma ou através de um computador via conexão USB (Universal Serial Bus). Encontramos relatos de implementações de dispositivos leitores de chaves óticas usando placas Arduino $7,9,14,15$, porém, não encontramos, até a presente data, uma descrição completa de um sistema de hardware e software que utilize todo o potencial das entradas analógicas da Arduino conjugadas com sensores de fácil montagem.

Em nossa proposta, até seis chaves óticas podem ser conectadas às entradas analógicas de uma placa Arduino Uno para aquisição quase simultânea dos sinais temporais, os quais são transmitidos, mostrados e tratados por um software dedicado para interfacear com esse sistema. As chaves óticas são compostas pelo tradicional par de componentes optoeletrônicos: LED infravermelho (modelo TIL32) e fototransistor (TIL78).

A escolha do uso da placa Arduino em conjunto com um computador (o qual pode ser um notebook ou similar) possibilita a combinação das potencialidades de um microcontrolador (ATmega328P no caso da Arduino) para aquisição e pré-tratamento de dados juntamente com os recursos de software operados por um microprocessador de um notebook, por exemplo. Com isso, é possível a aquisição de sinais analógicos de até seis chaves óticas independentes (no caso do modelo Arduino Uno) e o tratamento e amostragem dos sinais com ferramentas gráficas sofisticadas e de acesso livre. Como resultado, temos um sistema de aquisição sofisticado e versátil sem a necessidade de confecção de hardware avançado.

O sistema de hardware que descrevemos a seguir é composto pela placa de circuito impresso (chamada de shield que descrevemos na seção 2.2 responsável pela conexão entre a placa Arduino e os seis conectores das chaves óticas, e pelo recipiente de acondicionamento do par LED/fototransistor que compõe cada chave ótica. Também descrevemos e disponibilizamos o software que deve ser carregado na placa Arduino (escrito em linguagem similar a $\mathrm{C} / \mathrm{C}++$ ) e o software de aquisição e manipulação dos dados recebidos da placa Arduino e operado em um computador (escrito em linguagem Python). Tanto os desenhos do diagrama do circuito e da placa de circuito impresso quanto os códigos dos softwares utilizados estão disponíveis em serviços online de compartilhamento de projetos 16, 17. Por simplicidade, adotamos a nomenclatura de "sistema photogate" ao sistema completo de hardware + software.

Por fim, exemplificamos o uso do sistema em três experimentos: (i) verificação do MRUV no deslocamento de uma esfera por um plano inclinado, (ii) aplicação da lei de Stokes para determinação da viscosidade de um fluido e (iii) determinação do período de um pêndulo simples.

\section{Descrição do hardware}

Apresentamos na Fig. 1 um fluxograma do sistema completo com o objetivo de facilitar a identificação de cada parte do projeto no decorrer de sua descrição em cada subseção.

\subsection{A placa Arduino}

A principal peça de hardware utilizada nesta proposta é a plataforma Arduino [18], a qual encontra-se extensamente descrita em vários âmbitos e possui diversas aplicações no ensino. As quatro principais vantagens em se usar esse sistema, em nossa opinião, são: (i) o conceito de hardware livre, que permite facilidade de comunicação e

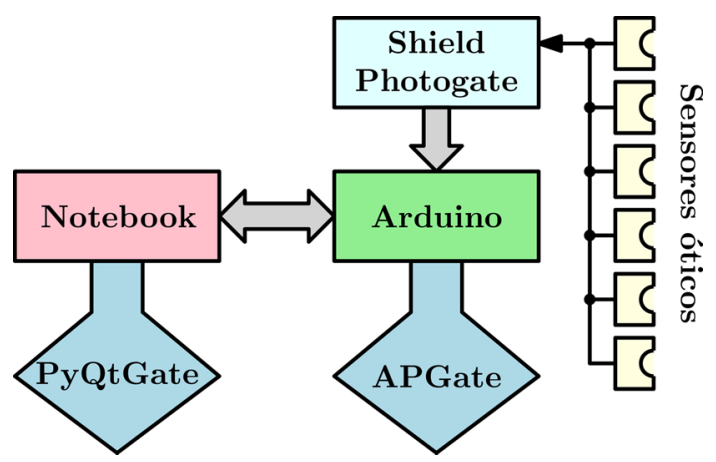

Figura 1: Fluxograma do sistema completo mostrando as relações entre as partes do hardware (retângulos) e as partes do software (losangos). As setas indicam o sentido da comunicação. 
de acesso ao produto, (ii) filosofia de introdução amigável aos conceitos de eletrônica e programação, permitindo que não especialistas desenvolvam projetos eletrônicos relevantes, (iii) incorporação de diferentes dispositivos avançados em uma única placa (microcontrolador, oscilador, reguladores de tensão, comunicação serial, filtros, design otimizado, etc.), poupando o usuário da necessidade de montar arranjos complexos e excessivamente técnicos e (iv) portabilidade da plataforma para diferentes projetos - através do conceito de shields, é possível acoplar hardware periférico à placa Arduino ao estilo plug and play.

O modelo de placa que utilizamos é a Arduino Uno mostrado na Fig. 2, pelo motivo de ser um dos mais difundidos, documentados e de mais baixo custo. Ainda assim, possui os recursos necessárias para este projeto: notadamente, seis entradas de sinal analógico.

Neste trabalho, o papel da placa Arduino é ler os sinais analógicos gerados por 6 portas óticas independentes, fazer a conversão analógica-digital desses sinais para variáveis de 10 bits e transferir esses valores para um notebook via comunicação USB. Uma vez transferidas para o computador, essas variáveis são expostas graficamente e parâmetros de amostragem são manipulados e registrados usando-se, para isso, uma interface gráfica específica descrita na seção 3.2

A Arduino Uno possui o microcontrolador ATmega328P com memória flash de $32 \mathrm{kB}$, na qual é gravado o código de controle, e memória SRAM de $2 \mathrm{kB}$ onde são manipuladas as variáveis em tempo de execução. Ou seja, é necessário gravar um código específico no microcontrolador para que ele execute os comandos/funções de interesse do projeto. No nosso caso, usamos a Arduino como um leitor dos sinais das portas óticas através das suas seis entradas analógicas (pinos A0 a A5) - os conectores de entrada/saída digitais não foram utilizados. Além desses, também utilizamos os pinos de $5 \mathrm{~V}$ e $0 \mathrm{~V}$ (GND) para alimentação dos LED's infravermelhos e fototransistores contidos nas portas óticas. A Fig. 3 contém as ligações as-

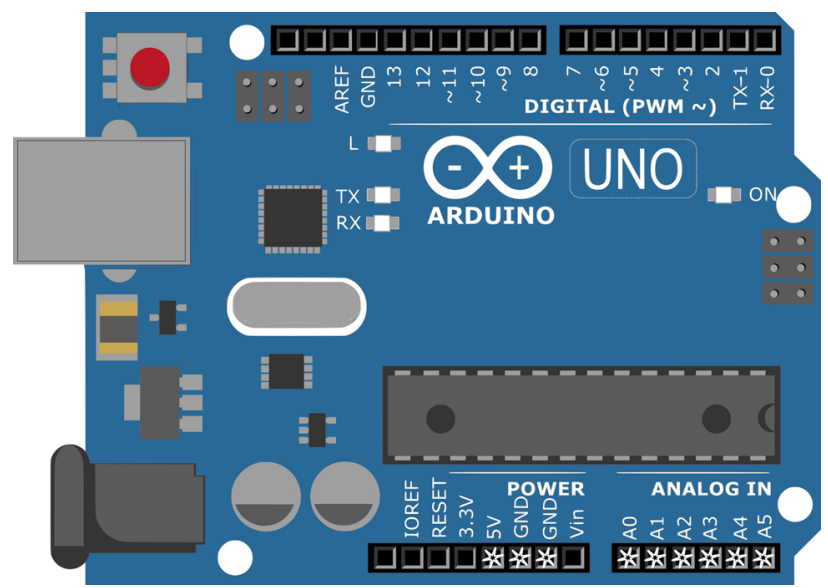

Figura 2: Desenho da placa Arduino Uno. Os pinos usados neste projeto estão marcados com asteriscos.

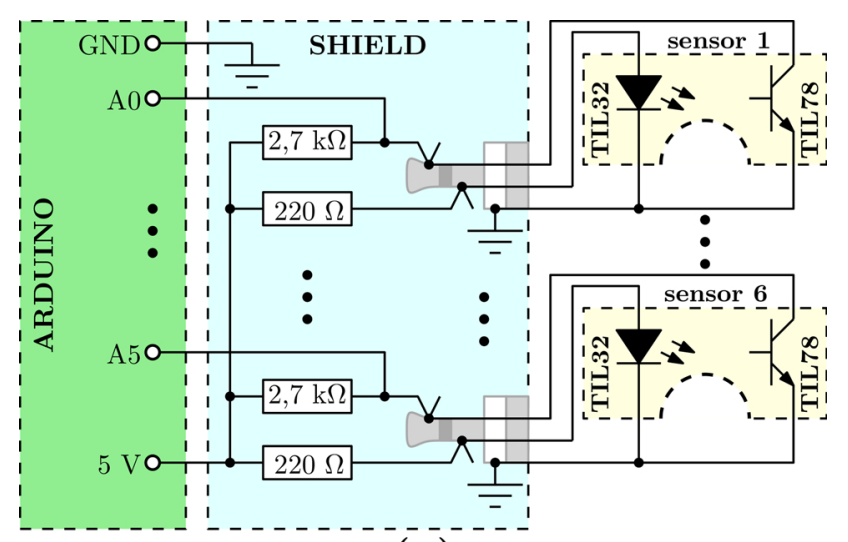

(a)

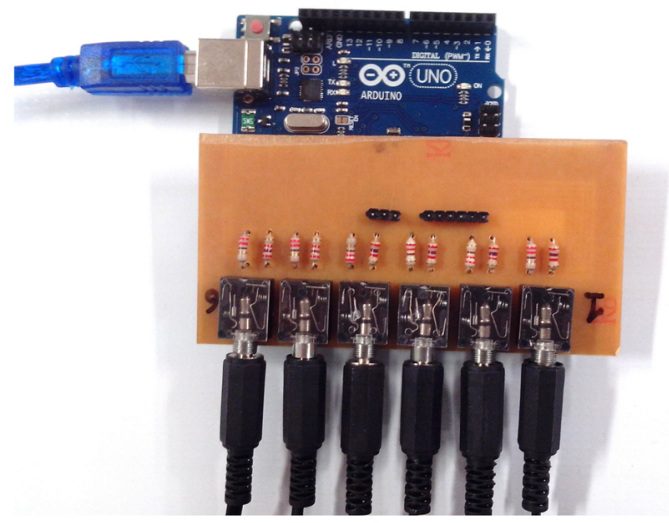

(b)

Figura 3: a) Diagrama do circuito eletrônico do sistema photogate. b) Foto do shield encaixado na Arduino e conectado às seis chaves óticas.

sociadas à placa Arduino. O código de controle é descrito na seção 3.1

\subsection{O shield photogate}

Percebe-se na Fig. 2 que os pinos de entrada e saída estão dispostos em linha via barra de conectores fêmea. A maneira mais direta de fazer a ligação elétrica com a Arduino é acoplando cabos flexíveis avulsos aos conectores de interesse. Entretanto, essa abordagem não é prática quando vários conectores são utilizados simultaneamente ou quando uma mesma placa Arduino é utilizada em diferentes projetos. Uma forma mais robusta e rápida de conexão é via o conceito de shield em que um circuito específico é construído sobre uma placa de circuito impresso, na qual são soldados conectores macho que se encaixarão em grupo nos conectores fêmea da Arduino. Além disso, o shield possui a eletrônica auxiliar necessária para o projeto, bem como os conectores para os sensores externos (portas óticas neste caso). Existem exemplos de shields comerciais aplicados ao ensino de física [19,20], sendo que desenvolvemos um modelo específico cujo circuito eletrônico é exposto na Fig. 3 Ele é composto por uma parte que contém o par avulso LED/fototransistor 
e outra que contém os resistores e pinos que compõe o shield.

A detecção de luz é realizada pelo fototransistor que é configurado no modo emissor comum. Ao iluminar a sua junção emissor-base com o LED infravermelho, gera-se uma corrente de emissor que varia com a intensidade da luz [21]. Para uma iluminação suficientemente intensa, o potencial no coletor, o qual está ligado a uma entrada analógica da Arduino, é zero. No momento em que a luz é interrompida, cessa-se a corrente de emissor e o potencial no coletor iguala-se à voltagem de alimentação de $5 \mathrm{~V}$. Outras configurações eletrônicas também podem ser utilizadas 8]. Como se vê no circuito, são necessários dois resistores: um de $220 \Omega$ para limitar a corrente do LED infravermelho e outro de $2,7 \mathrm{k} \Omega$ para limitar a corrente do coletor do fototransistor e dividir a tensão com o coletor-emissor - existe certa flexibilidade na escolha desses valores.

Os componentes óticos (par LED/fototransistor) não são soldados ao shield, mas conectados de forma a termos a liberdade de acoplar/desacoplar um número de sensores conveniente ao experimento sendo realizado - limitado a seis.

Tornamos público tanto o diagrama do circuito da Fig. 3 quanto o desenho da placa de circuito impresso que compõe o shield. Ambos podem ser copiados e editados no site EasyEDA [16], que é uma ferramenta online gratuita para criação, simulação e compartilhamento de projetos eletrônicos. Com isso, qualquer pessoa pode copiar os diagramas e desenhos aqui descritos; mas mais do que isso: editá-los e adaptá-los aos seus próprios interesses ou tomá-los como ponto de partida para outros projetos. A plataforma EasyEDA é bastante amigável e possui a vantagem de rodar diretamente no navegador de internet, sem necessidade de instalação de arquivos específicos. Outras opções online e offline de softwares livres para desenho/simulação de circuitos também existem [22].

\subsection{Montagem dos sensores}

Para acondicionar os pares LED/fototransistor que compõe as portas óticas, desenvolvemos o recipiente ilustrado na Fig. 4. Cada recipiente é construído a partir de uma placa quadrada de MDF (Medium Density Fiberboard) de $8,5 \mathrm{~cm}$ de lado e $9 \mathrm{~mm}$ de espessura. Nela é feito um furo de $1,25 \mathrm{~cm}$ de diâmetro por onde passa a haste de suporte e é recortado um semicírculo de $6,5 \mathrm{~cm}$ de diâmetro por onde passam os obstáculos a serem medidos pela porta ótica. Para produzir o furo é usada uma broca comum para madeira e para o semicírculo é usada uma serra copo.

Para acondicionar o LED e o fototransistor, é feito um furo passante paralelo e próximo à borda da placa interrompida pelo recorte em semicírculo, de forma que os dois componentes fiquem faceados e alinhados.

Adicionalmente, furos passantes diagonais à placa são utilizados para conduzir e fixar os cabos elétricos.

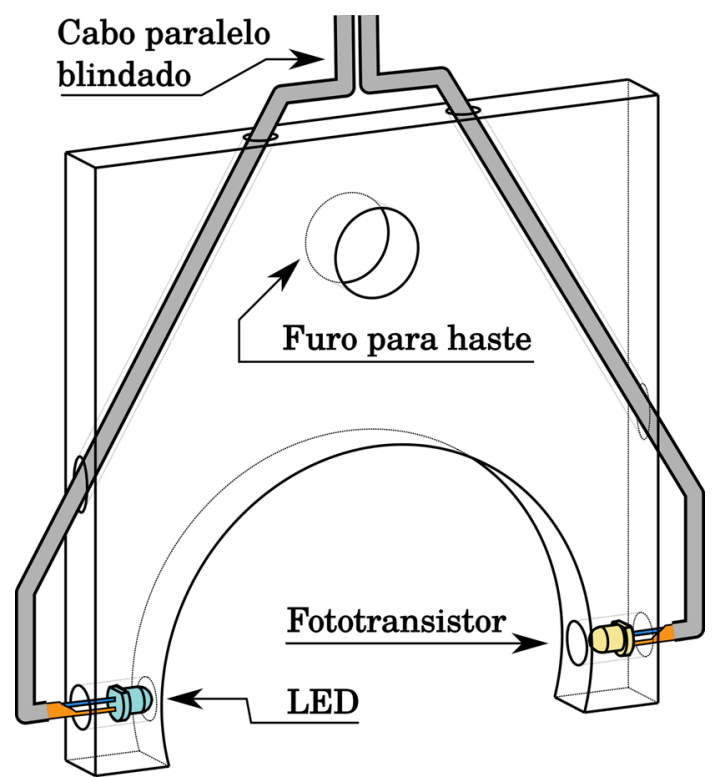

Figura 4: Desenho do recipiente do sistema photogate.

O cabo elétrico utilizado é do tipo estéreo paralelo, em que cada via possui uma blindagem independente, a qual é utilizada como referência de $0 \mathrm{~V}$ em nosso projeto.

Esse é um arranjo robusto, de muito baixo custo e que permite a produção das peças em série sem necessidade de ferramentas profissionais.

\section{Descrição do software}

Para adquirir e processar os dados, foram usados dois códigos de programação. Um escrito em linguagem $\mathrm{C}$ que é carregado no microcontrolador da Arduino e outro em linguagem Python com a biblioteca PyQt para processamento dos dados e desenvolvimento da interface gráfica a ser usada no computador. Chamamos de APGate (Arduino PhotoGate) o código em C e de PyQtGate o código em Python. Ambos são descritos a seguir e seus códigos fonte originais podem ser obtidos no repositório GitHub [17].

\subsection{O código APGate para Arduino}

O APGate é constituído por duas partes: (i) protocolo de identificação/comunicação entre o APGate e o PyQtGate e (ii) leitura e envio dos sinais dos seis sensores óticos para o computador via interface USB.

Foge ao escopo deste artigo fazer uma descrição pormenorizada da sintaxe e funcionamento do código. Para esse detalhamento, recomendamos o estudo dos diversos tutoriais disponíveis no site oficial da Arduino ou nos fóruns online pertinentes. Para facilitar o entendimento, o código encontra-se comentado em 17].

Ainda assim, vale destacar um conjunto de comandos especialmente importantes a este projeto que determinam a velocidade de leitura dos sinais analógicos dos senso- 
res óticos e que não estão documentados nos tutoriais iniciais. A velocidade de leitura dos pinos analógicos é determinada pela frequência de clock do microcontrolador. Para obter a resolução máxima de 10 bits da voltagem que está sendo lida (5 V divididos em 1024 níveis), o microcontrolador aplica um fator de divisão igual a 128 . Por exemplo, com esse fator, se a frequência do sistema do microcontrolador for de $16 \mathrm{MHz}$, a frequência efetiva do conversor analógico-digital será de $125 \mathrm{kHz}$. Como a leitura dos seis sensores óticos é feita sequencialmente, queremos que ela seja a mais rápida possível de forma a podermos considerar essas leituras como sendo aproximadamente simultâneas. Utilizamos um fator de divisão de 16 (clock efetivo de $1 \mathrm{MHz}$ ), o qual apresentou um bom compromisso entre resolução e velocidade de aquisição.

Por outro lado, em nosso código, o parâmetro que determina o intervalo de aquisição entre um conjunto de medidas dos seis sensores e o conjunto de medidas seguinte é a velocidade de comunicação serial entre a Arduino e o computador. A velocidade padrão utilizada na Arduino Uno é de 9600 bits por segundo (bps), podendo ser alterada para no máximo 115200 bps. Com essa velocidade máxima, o nosso código apresentou um intervalo de tempo entre conjuntos seguidos de aquisição igual à aproximadamente $1 \mathrm{~ms}$. Acreditamos que esse intervalo é suficientemente pequeno para a maioria das aplicações das portas óticas para o ensino de física, conforme ilustramos na seção 4

\subsection{O programa PyQtGate para visualização e manipulação dos dados}

Para escrever a interface gráfica de visualização e manipulação dos dados, utilizamos a linguagem Python 23] juntamente com a biblioteca PyQt 24]. A linguagem Python enquadra-se na categoria de alto nível e conta com uma extensa rede de colaboradores e programadores. Destaca-se pela facilidade de programação de códigos simples e avançados, pela diversidade de bibliotecas desenvolvidas para os mais diversos fins e pela extensa documentação. A biblioteca PyQt permite o uso, no ambiente Python, dos objetos da plataforma Qt a qual é utilizada no desenvolvimento de interfaces gráficas (janelas, botões, menu de arquivos, etc.) para programas em $\mathrm{C} / \mathrm{C}++$ e outras linguagens. Para isso, existe a ferramenta Qt Designer 25] em que os diferentes objetos gráficos são dispostos e conectados de acordo com o projeto em questão, sendo gerado um código a eles associados para ser usado e manipulado na linguagem de interesse (Python, no nosso caso).

Assim como o código APGate, não é nosso objetivo descrever aqui os detalhes do programa PyQtGate. Apenas descreveremos suas funcionalidades com o objetivo de ilustrar o potencial das abordagens propostas neste trabalho. O código fonte original comentado pode ser obtido em [17.
A Fig. 5 apresenta a interface do programa. As abas horizontais correspondem às seguintes opções: (i) "Mostrador": em que as formas de onda dos sinais dos sensores óticos são mostradas em função do tempo, (ii) "Arquivo": em que as formas de onda adquiridas e/ou os tempos de disparo podem ser gravados e carregados, (iii) "Serial": para iniciar ou interromper a comunicação com o código APGate na Arduino e (iv) "Sobre": que contém informações sobre os programas e procedimentos necessários para implementar esta proposta de portas óticas.

Na base da janela da interface encontra-se o botão de Iniciar/Parar a aquisição dos dados dos sensores óticos ao lado da informação de status da aquisição.

A aba "Mostrador" contém duas abas verticais para apresentação das formas de onda separadas ou juntas no mesmo gráfico. A Fig. 5 a) apresenta a opção "Juntos". Nela, as formas de onda dos sensores óticos habilitados nas caixas de seleção à direita são mostradas no mesmo gráfico juntamente com os níveis de disparo para contagem de tempo. Os níveis de disparo são representados por uma linha tracejada horizontal da mesma cor que a forma de onda do respectivo sensor. Tendo lido um

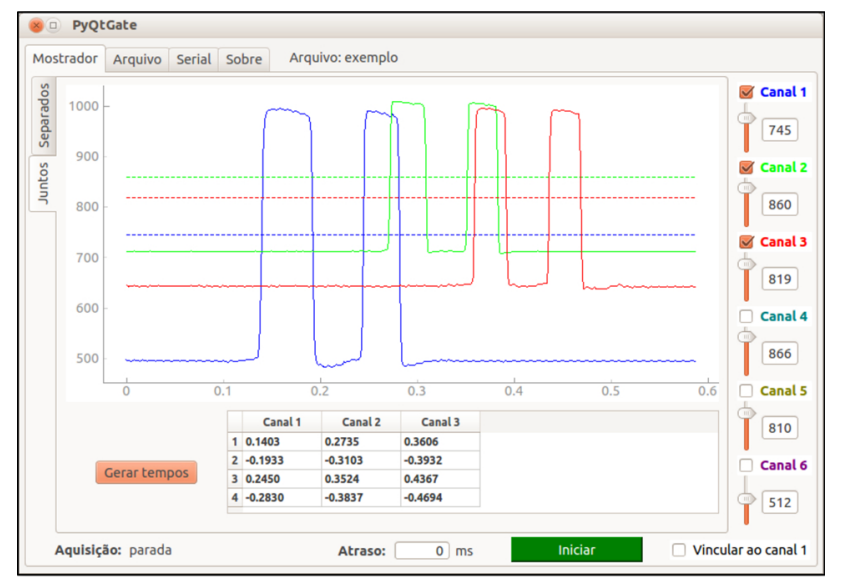

(a)

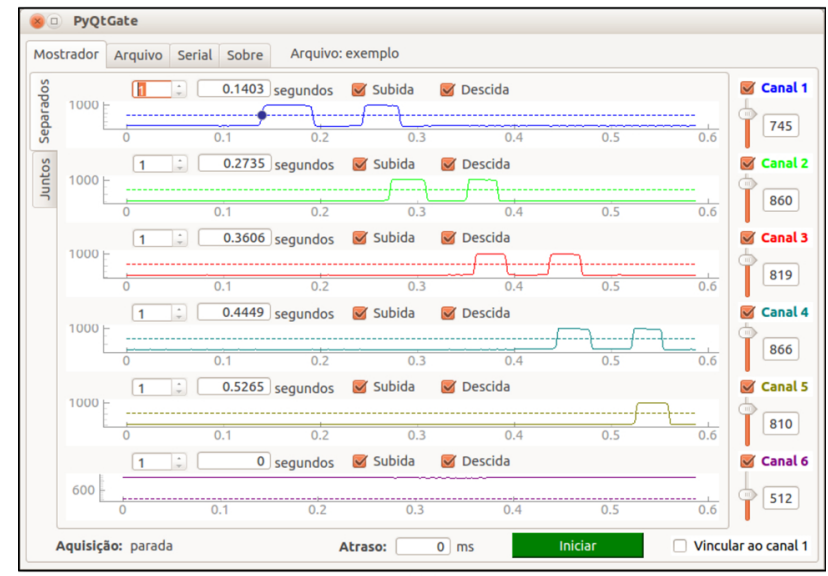

(b)

Figura 5: Interfaces do programa PyQtGate em que os sinais das portas óticas são mostrados a) juntos e b) separados. 
conjunto de formas de onda, ao clicar no botão "Gerar tempos" é gerada a tabela à direita desse botão com os instantes de tempo em que a forma de onda cruza os respectivos níveis de disparo. Adotamos a convenção de tempo positivo quando o disparo ocorre na subida do sinal e de tempo negativo quando ocorre na descida. Não há ambiguidade nessa escolha, uma vez que o instante de tempo inicial da aquisição é sempre fixado em zero. No exemplo da Fig. 5 a), para o canal 1, temos dois disparos na subida em $t=0,1403 \mathrm{~s}$ e $t=0,2450 \mathrm{~s}$ intercalados por dois disparos na descida em $t=0,1933 \mathrm{~s}$ e $t=0,2830 \mathrm{~s}$. O nível de disparo de cada canal pode ser ajustado independentemente pelas barras de rolagem à direita do gráfico ou ajustados concomitantemente mexendo-se apenas no canal 1 caso a opção "Vincular ao canal 1" esteja marcada.

A Fig. 5b) apresenta a aba "Separados" do "Mostrador". Ela mostra as mesmas formas de onda da aba "Juntos" separadas umas das outras. Os tempos de disparo são mostrados em displays separados e há a opção de mostrar somente os tempos de subida, de descida ou ambos. Os diferentes tempos de cada canal são mostrados individualmente ao se varrer a caixa de rolagem à esquerda do respectivo display de tempo.

Como dissemos, uma das vantagens de se trabalhar com a linguagem Python são as diversas bibliotecas que permitem o uso de recursos sofisticados sem a necessidade de escrita de códigos avançados. Esse é um aspecto em comum com a filosofia da Arduino e, por isso, acreditamos que essa combinação específica de hardware e software seja bem produtiva. Podemos citar, por exemplo, as bibliotecas PySerial 26] e PyQtGraph [27] utilizadas em nosso programa. A primeira disponibiliza comandos amigáveis para se estabelecer comunicação serial com diversos dispositivos e a segunda apresenta ferramentas gráficas avançadas para visualização e tratamento de dados como aqueles vistos na Fig. 5 .

\section{Exemplos de aplicação}

Descrevemos a seguir três exemplos de aplicação do sistema photogate a experimentos clássicos de ensino de física. O intuito é mostrar as vantagens referentes à visualização gráfica das formas de onda dos sensores óticos, ao número grande de canais quase simultâneos, à capacidade de ajuste dos níveis de disparo e à utilização de ferramentas matemáticas para análise dos gráficos.

\subsection{MRUV no plano inclinado}

Demonstramos aqui o experimento de movimento retilíneo uniformemente variado (MRUV) associado à rolagem de uma esfera por um plano inclinado submetida à ação da gravidade. Com o nosso sistema photogate podemos medir tanto a posição quanto a velocidade instantânea da esfera em função do tempo para seis pontos distintos da trajetória. É possível mostrar que, despre- zando a dissipação de energia mecânica, uma esfera rolando sem deslizar por um plano inclinado de $\theta$ graus em relação à horizontal executa MRUV com aceleração linear dada por:

$$
a=\frac{5}{7} g \operatorname{sen} \theta,
$$

em que $g$ é a magnitude da aceleração gravitacional.

A Fig. 6] apresenta a foto do arranjo do plano inclinado e da disposição das seis chaves óticas ao longo da trajetória da esfera. Os fototransistores das chaves óticas foram alinhados e centralizados rente a trajetória da esfera e dispostos equidistantes entre si por aproximadamente $8 \mathrm{~cm}$. A inclinação do plano foi de $2,4^{\mathrm{O}} \mathrm{e}$ o diâmetro da esfera foi de $2,54 \mathrm{~cm}$. Os materiais da esfera e do plano inclinado foram, respectivamente, aço e alumínio.

As formas de onda registradas pelo sistema photogate encontram-se na Fig. 7. Escolhemos como nível de disparo a meia altura entre a linha de base e a amplitude máxima de cada canal. Com esses dados, aproximamos

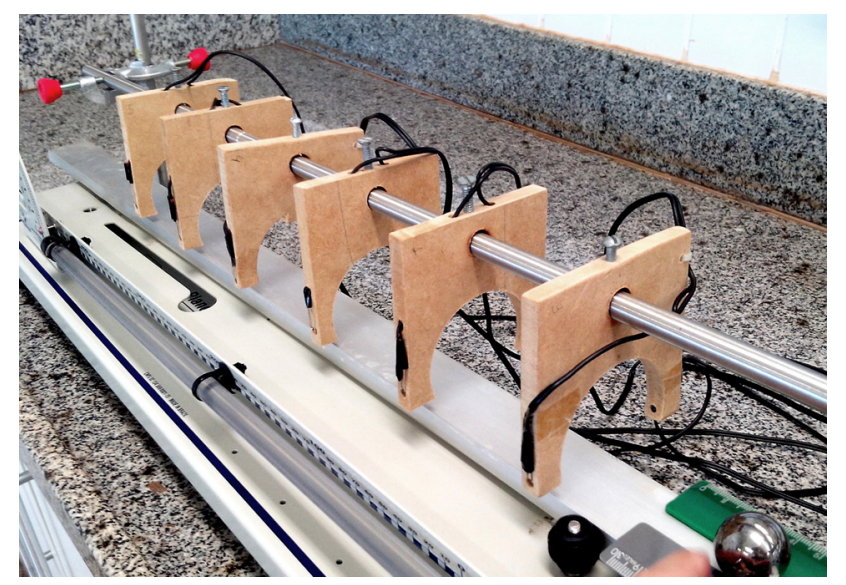

Figura 6: Foto do arranjo experimental do plano inclinado e a disposição das seis chaves óticas ao longo da trajetória da esfera.

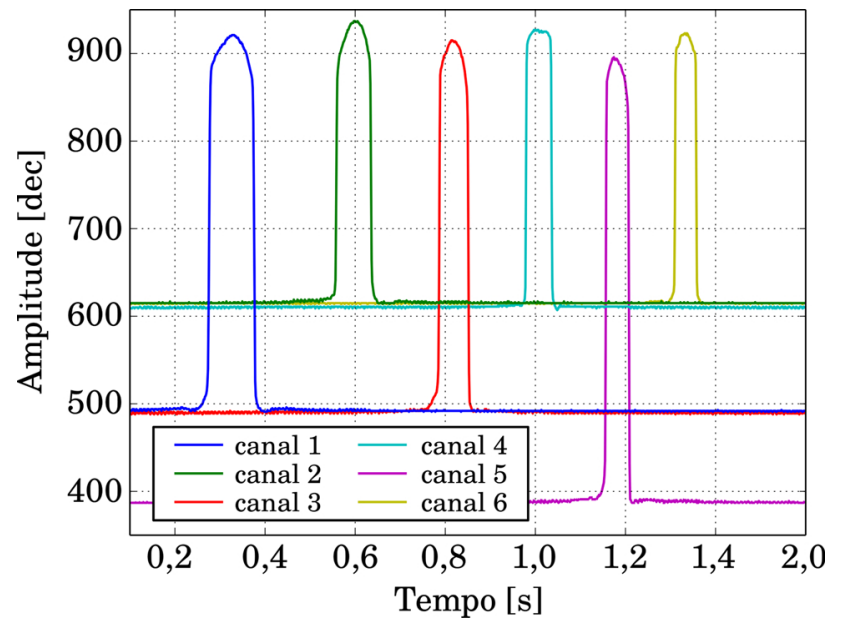

Figura 7: Formas de onda registradas pelo sistema photogate referentes ao movimento da esfera no plano inclinado para um mesmo lançamento. 
os instantes de tempo $t$ em que o centro da bola passou pela posição $x$ de cada sensor ótico tomando-se a média entre os tempos de subida e descida da respectiva forma de onda. Além disso, foi possível calcular a velocidade instantânea $v$ em cada instante $t$ dividindo-se o diâmetro da esfera pelo intervalo de tempo entre os tempos de subida e descida. A Fig. 8 apresenta os gráficos com os dados experimentais de $x(t)$ e $v(t)$, bem como as respectivas curvas teóricas ajustadas.

Foi possível obter o valor de $g$ a partir do coeficiente quadrático da curva ajustada para $x(t)$ para cinco lançamentos distintos da esfera, resultando em $g=$ $(9,49 \pm 0,03) \mathrm{m} / \mathrm{s}^{2}$. Vemos que, apesar dos lançamentos serem independentes - velocidades e posições iniciais distintas - o desvio correspondeu a apenas $0,3 \%$ do valor médio. $\mathrm{O}$ valor de $g$ também pôde ser obtido a partir do coeficiente angular da reta ajusta para $v(t)$ para os cinco lançamentos, resultando em $g=(9,7 \pm 0,1) \mathrm{m} / \mathrm{s}^{2}$. Portanto, além da tendência dos pontos experimentais estarem em grande acordo com o esperado para o MRUV, os valores de $g$ obtidos pelos dois métodos diferem por apenas $2 \%$, fortalecendo a qualidade das medidas realizadas com o sistema photogate. $\mathrm{O}$ valor esperado para $g$ na localidade do experimento (220 $18^{\prime} 54^{\prime \prime} \mathrm{S} ; 47^{\circ} 22^{\prime} 53$ " $\mathrm{O} ; 704 \mathrm{~m}$ ) é de $9,7856 \mathrm{~m} / \mathrm{s}^{2}$ 28. A principal suspeita para a subestimativa dos valores medidos recai sobre a dissipação de energia mecânica devido às irregularidades e interações de contato da esfera com a superfície do plano inclinado.

Em resumo, vemos neste exemplo que os seis canais analógicos auxiliam a visualizar as propriedades cinemáticas do MRUV ao amostrar, em seis posições e em seis instantes de tempo, um único lançamento da esfera, além de permitir inferir o intervalo de obstrução pela passagem da

(a)

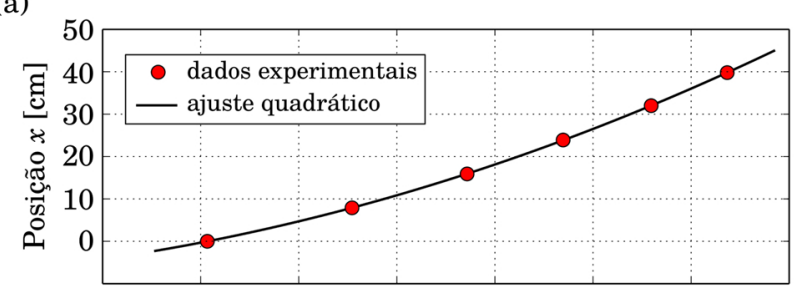

(b)

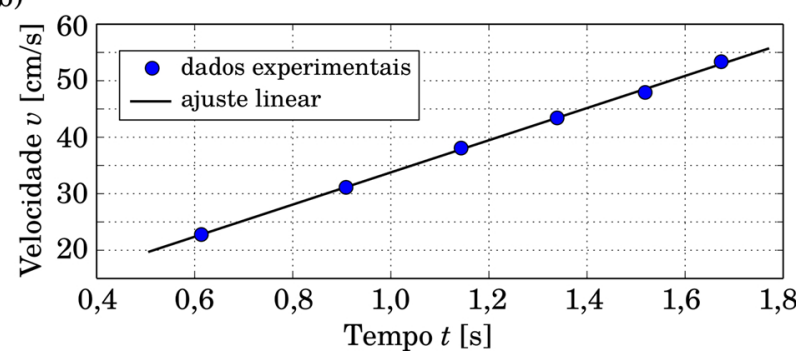

Figura 8: Gráficos do experimento do plano inclinado. a) Posição e b) velocidade instantânea da esfera em função do tempo. As barras de erros são menores que o tamanho dos símbolos. esfera e, consequentemente, sua velocidade instantânea (no caso do MRUV).

\subsection{Lei de Stokes}

Para ilustrar um exemplo de aplicação no estudo de fluidos, apresentamos os resultados do experimento envolvendo o movimento de queda de esferas de aço em um tubo com glicerina com o objetivo de explorar as propriedades do escoamento de fluidos viscosos e a aplicação da lei de Stokes.

Esse também é um experimento clássico nos laboratórios de ensino de física em que, no caso de instrumentação simples, o estudante solta uma esfera metálica em um tubo comprido contendo um líquido de viscosidade $\eta$ - geralmente glicerina - e, utilizando um cronômetro, faz a aferição do tempo de queda através de uma altura pré-definida. O experimento é então repetido para diferentes alturas e esferas de diferentes diâmetros. Dos dados obtidos é possível inferir se as esferas alcançaram velocidade terminal $v$ e obter o gráfico dessa velocidade em função do diâmetro $d$ de cada esfera.

No caso de escoamento a baixas velocidades, espera-se que a força de arrasto de Stokes, $F=3 \pi d \eta v$, se aplique com um fator de correção $f(d)$ e, consequentemente:

$$
v=\frac{g\left(\rho_{e}-\rho_{f}\right)}{18 \eta} d^{2} f(d),
$$

em que $\rho_{e}$ e $\rho_{f}$ são, respectivamente, as densidades da esfera e do fluido e $g$ é a magnitude da aceleração gravitacional. O fator de correção $f(d)$ leva em conta o confinamento do movimento e depende da relação entre o tamanho da esfera e a geometria do tubo. O fator de correção que melhor descreveu nossos dados foi a combinação da fórmula de Faxen para a razão $\frac{d}{D}$ e o fator de Ladenburg para a razão $\frac{d}{H} 29$ dado por:

$$
f(d)=\frac{1-2,104 \frac{d}{D}+2,09\left(\frac{d}{D}\right)^{3}}{1+1,65 \frac{d}{H}},
$$

em que $D$ e $H$ são, respectivamente, o diâmetro interno do tubo e a altura da coluna de líquido.

Normalmente, é utilizado para esse experimento um tubo de vidro com comprimento superior a $70 \mathrm{~cm}$. Uma das justificativas para o uso de um tubo comprido é para que o tempo de queda da esfera no líquido seja grande relativamente ao erro no tempo associado ao acionamento manual do cronômetro. Utilizando-se o sistema photogate, esse erro é bastante minimizado e, com isso, podemos usar tubos mais curtos. Em nossa demonstração, utilizamos uma proveta plástica translúcida com graduação máxima de $250 \mathrm{ml}$ e diâmetro interno $D=3,4 \mathrm{~cm}$, em que a coluna de glicerina tinha altura $H=31,5 \mathrm{~cm}$. Essa escolha viabiliza a realização de um maior número de experimentos, pois utiliza menos glicerina e reduz enormemente o custo do tubo utilizado. Foram usados seis diâmetros de esfera diferentes: 2,95, 4,45, 6,30, 7,15, 7,90 e $9,50 \mathrm{~mm}$. 
A Fig. 9 apresenta a foto do arranjo das seis chaves óticas ao longo da proveta com glicerina, as quais foram alinhadas com o auxílio de um prumo. A separação entre os sensores foi de aproximadamente $5,0 \mathrm{~cm}$. As formas de onda registradas pelo sistema photogate para o movimento de queda de uma esfera de $2,95 \mathrm{~mm}$ de diâmetro encontram-se na Fig. 10. Devido ao reduzido diâmetro das esferas e à distância das suas trajetória aos fototransistores, não foi possível obter medidas confiáveis de velocidade instantânea usando o intervalo de tempo de obstrução, conforme descrito na seção 4.1. De fato, para sistemas de portas óticas tradicionais - que não disponibilizam a forma de onda do sinal do sensor ótico - é desafiador fazer com que esferas pequenas disparem as portas óticas. Com a visualização do sinal, podemos ter a noção de quão bom está o alinhamento das diferentes chaves óticas e podemos ajustar os níveis de disparo para as diferentes sensibilidades dos fototransistores, as quais não serão as mesmas devido a ajustes sutis em seus alinhamentos.

A Fig. 11 a) apresenta o gráfico da altura em função do tempo para as seis esferas diferentes. Dos ajustes lineares, percebe-se que a condição de velocidade terminal foi bem satisfeita, mesmo usando-se uma altura de coluna de líquido significativamente mais curta do que na abordagem tradicional com tubo de vidro. Dos

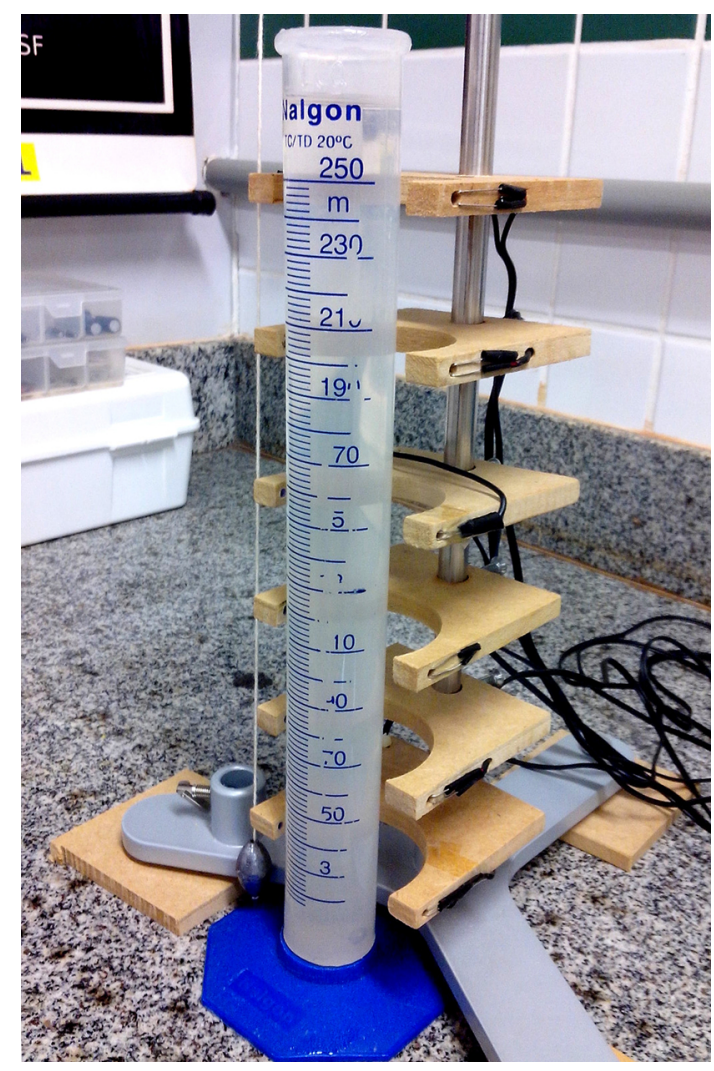

Figura 9: Arranjo experimental do viscosímetro com as seis chaves óticas aprumadas com a direção de queda das esferas de aço.

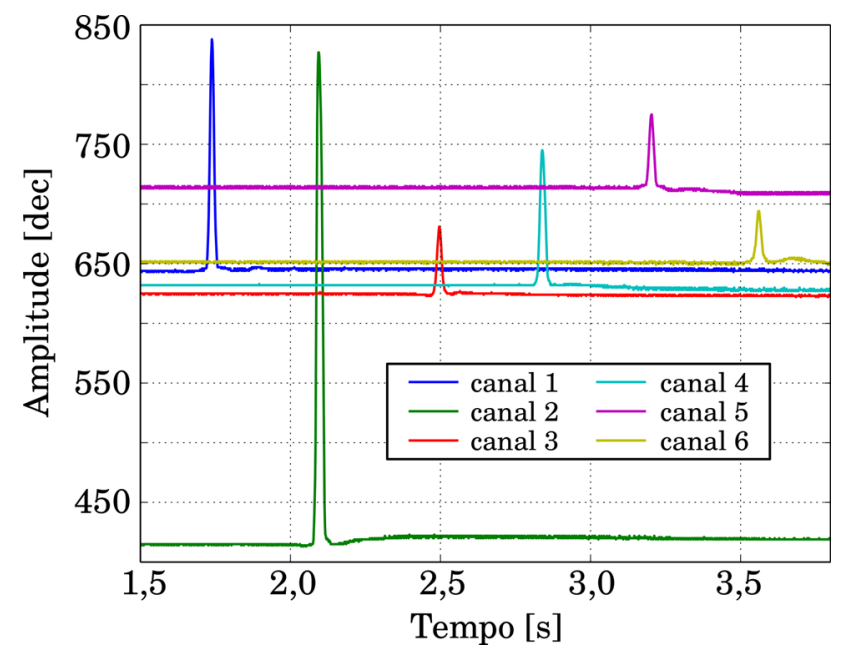

Figura 10: Formas de onda registradas pelo sistema photogate referentes ao movimento de queda em glicerina da esfera de $2,95 \mathrm{~mm}$ de diâmetro para um mesmo lançamento.
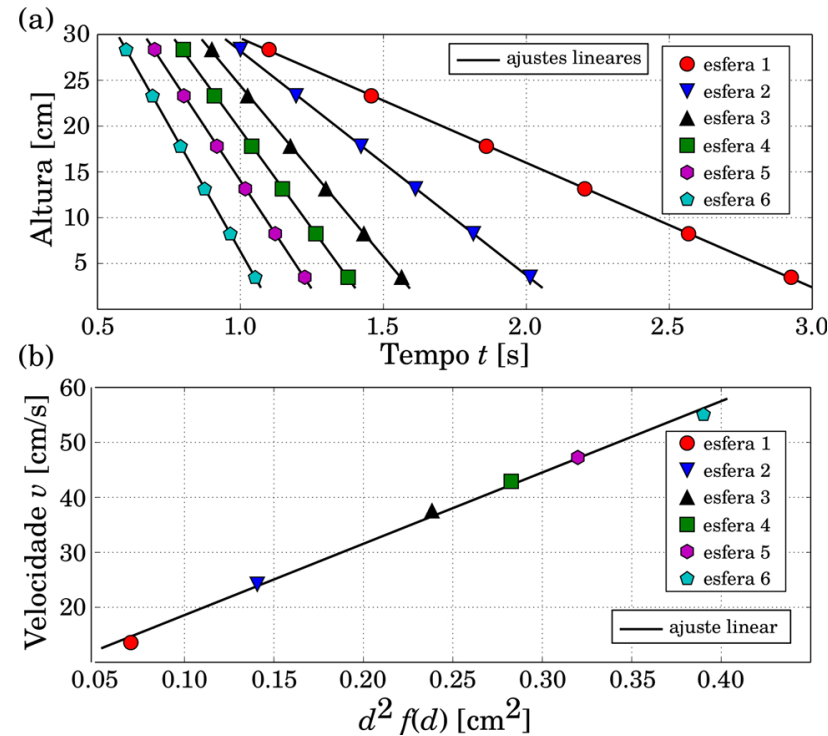

Figura 11: Gráficos do experimento de viscosidade. a) Altura em função do tempo, em que os instantes iniciais foram deslocados para não haver sobreposição dos pontos, e b) velocidade terminal em função do diâmetro da esfera com fator de correção. A numeração das esferas está em ordem crescente de diâmetro. As barras de erros são menores que o tamanho dos símbolos.

coeficientes angulares das retas ajustadas foi possível construir o gráfico da velocidade terminal em função do diâmetro ao quadrado corrigido, $d^{2} f(d)$, para as seis esferas, que se encontra na Fig. 11b). Do coeficiente angular do ajuste linear desse gráfico, obtivemos o valor de viscosidade da glicerina através da Eq. (2) que resultou em $\eta=(0,278 \pm 0,001)$ Pa.s. Os valores medidos para as densidades das esferas e da glicerina foram $\rho_{e}=(7850 \pm 10) \mathrm{kg} / \mathrm{m}^{3}$ e $\rho_{f}=(1220 \pm 10) \mathrm{kg} / \mathrm{m}^{3}$. Adota$\operatorname{mos} g=9,7856 \mathrm{~m} / \mathrm{s}^{2}$, conforme exposto na seção 4.1 A temperatura da glicerina era de aproximadamente $23{ }^{\circ} \mathrm{C}$. Comparado com o valor tabelado para a densidade da 
glicerina nessa temperatura [30] inferimos uma mistura, em massa, de $87 \%$ de glicerina e $13 \%$ de água. A viscosidade esperada para a glicerina nessa concentração e temperatura é de 0,123 Pa.s [30], valor bastante aquém do obtido experimentalmente.

Existem fatores críticos para a obtenção de valores exatos de $\eta$ por este método [31]. O mais importante em nosso experimento foram os altos números de Reynolds $\left(R_{e}\right)$ envolvidos: $2<R_{e}<23$. Para que a lei de Stokes se aplique é necessário que o fluxo seja laminar em torno da esfera, o que demanda $R_{e} \ll 1$. Algumas formas de diminuir $R_{e}$ incluem o uso de uma glicerina mais pura, que aumenta grandemente sua viscosidade e diminui a velocidade terminal das esferas, e o uso de esferas com menor diâmetro.

Esse mesmo experimento foi realizado usando um tubo de vidro transparente com diâmetro de $5,0 \mathrm{~cm}$ e altura da coluna de glicerina igual a $70 \mathrm{~cm}$, cujos resultados não estão mostrados aqui. Não houve diferença significativa no comportamento dos dados experimentais e nos valores encontrados para a viscosidade.

Percebe-se que a baixa exatidão obtida para o valor experimental da viscosidade da glicerina foi devida aos materiais utilizados, sendo que a qualidade dos resultados gerados pelo sistema photogate possibilitou pôr à prova os modelos adotados.

\subsection{Pêndulo simples}

Como última aplicação, ilustramos a aferição do período de oscilação de um pêndulo simples e, por conseguinte, a magnitude da aceleração gravitacional. O pêndulo era constituído por uma massa esférica de aço de $2,54 \mathrm{~cm}$ de diâmetro suspensa por um fio de algodão com densidade linear de aproximadamente $0,09 \mathrm{~g} / \mathrm{m}$. A distância do ponto de suspensão ao centro da esfera foi de $(157,4 \pm$ $0,2) \mathrm{cm}$. Nesse caso, foi usada somente uma chave ótica posicionada no ponto de equilíbrio da massa esférica.

A Fig. 12.a) apresenta a forma de onda do sensor ótico obtida pelas sucessivas passagens do pêndulo. Para inferir o período, podemos tomar a média dos vários intervalos de subida ou de descida. Entretanto, a biblioteca PyQtGraph, discutida na seção 3.2 possui o recurso de Transformada de Fourier Discreta (TFD) acessível diretamente pela janela gráfica, bastando clicar com o botão direito sobre o gráfico para implementá-la. Na Fig. 12 b) expomos a TFD do sinal temporal da Fig. 12.a). Como o sinal temporal é formado por uma sucessão de formas de onda quase quadradas, a TFD contém um grande número de harmônicos da frequência fundamental, a qual corresponde à frequência de oscilação do pêndulo. Das propriedades da transformada de Fourier, quanto maior o tempo de aquisição, maior a resolução no domínio da frequência, aumentando, portanto, a precisão na determinação do período. O intervalo de tempo de aquisição do sinal foi de aproximadamente $306 \mathrm{~s}$, correspondendo a 121 oscilações. (a)

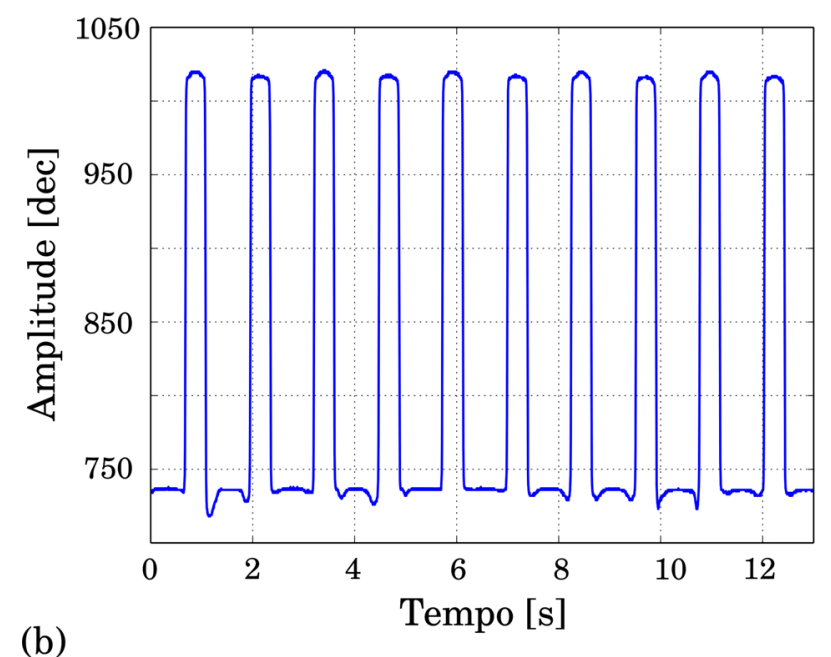

(b)

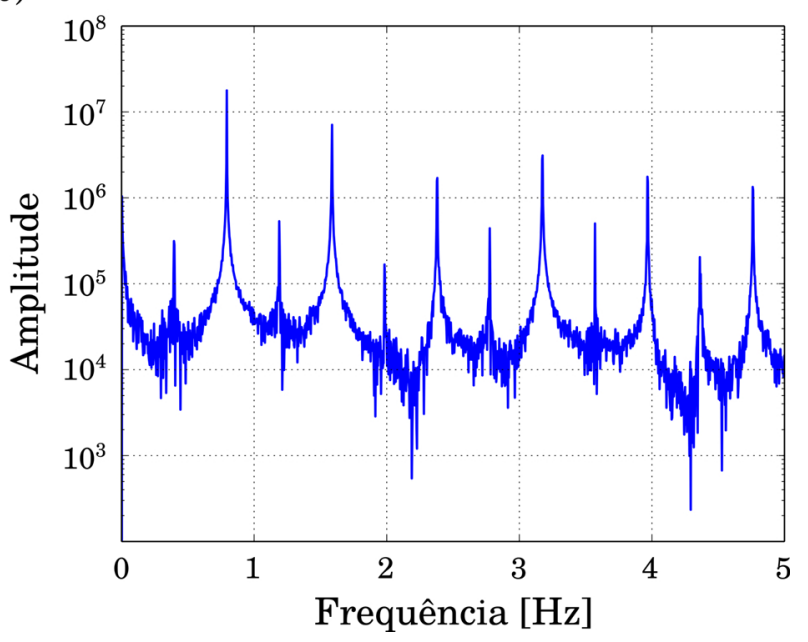

Figura 12: Formas de onda do pêndulo. a) Amplitude em função do tempo para as cinco primeiras oscilações e b) Amplitude espectral em função da frequência para os 12 primeiros harmônicos obtida da DFT do sinal temporal.

A TFD forneceu uma frequência de $\left(0,39683 \pm 7.10^{-5}\right) \mathrm{Hz}$, resultando em uma magnitude da aceleração gravitacional de $(9,79 \pm 0,01) \mathrm{m} / \mathrm{s}^{2}$. O erro associado a $g$ se deve quase inteiramente ao erro associado à aferição do comprimento do pêndulo. Esse resultado concorda com o valor esperado exposto na seção 4.1 .

\section{Conclusões}

Neste trabalho, apresentamos uma proposta de sistema de portas óticas usando plataformas de hardware e software livres que permitiram o desenvolvimento de um projeto com recursos razoavelmente sofisticados e que não necessitou de conhecimentos e procedimentos técnicos avançados para ser implementada. Apenas conhecimentos intermediários de eletrônica e programação foram usados. Além disso, a proposta se baseou no conceito de projetos compartilhados em que qualquer pessoa pode ter acesso aos códigos de programação e aos desenhos dos 
circuitos originais, podendo modificá-los conforme seus próprios interesses, bem como aprimorá-los para futuras implementações.

Achamos prudente alertar que os códigos de programação e os circuitos desenvolvidos neste trabalho carecem de uma arquitetura mais elaborada, sendo que os plenos recursos disponíveis nas diferentes plataformas são usados, muitas vezes, de forma um tanto rudimentar. Assim, por exemplo, para o código PyQtGate escrito em linguagem Python, usamos uma estrutura bastante procedural, sendo que para um código mais robusto e portável seria recomendável maior ênfase na programação orientada a objeto, que por sinal é parte essencial da linguagem Python. O mesmo vale para as otimizações do desenho do hardware, em que acessamos apenas a superfície dos recursos disponíveis nos softwares de desenhos eletrônicos. Ainda assim, acreditamos que a inteligibilidade e funcionalidade não foram significativamente afetadas por essas escolhas. Por acréscimo, isso reforça a nossa premissa de que conhecimentos avançados não são essenciais para o desenvolvimento deste nível de implementação.

Foram apresentados três exemplos de aplicação que ilustraram a versatilidade da proposta e a qualidade dos resultados que podem ser obtidos. Com isso, esperamos que ela contribua para o desenvolvimento e autonomia dos laboratórios de ensino de física ou de ciências através do uso de ferramentas acessíveis e atuais.

O objetivo do trabalho foi de agregar recursos aos laboratórios didáticos, mas não para servir de panaceia para o desenvolvimento de quaisquer atividades experimentais. Acreditamos que instrumentos de medidas e configurações experimentais mais básicas ainda são fundamentais para a formação dos estudantes, possibilitando um contato mais próximo com as grandezas físicas envolvidas e os procedimentos básicos do método científico. Além disso, é importante manter-se consciente sobre os processos subjacentes aos dispositivos de automação e digitalização para uma correta avaliação dos erros envolvidos e das limitações experimentais 32 .

Ainda assim, são vários os ganhos em se utilizar ferramentas digitais ou tecnológicas na aquisição de dados experimentais no âmbito dos laboratórios didáticos. Dentre eles, destacamos a possibilidade de se dedicar mais tempo à preparação cuidadosa dos arranjos experimentais em detrimento ao tempo usado para a realização de muitas repetições da mesma medida com o intuito de aumentar a sua precisão.

\section{Referências}

[1] Brasil. Secretaria de Educação Básica, Diretrizes curriculares nacionais da educação básica (MEC-SEB, Brasília, 2013).

[2] S. Kuznetsov e E. Paulos, in Proceedings of the 6th Nordic Conference on Human-Computer Interaction: Extending Boundaries, Reykjavik, 2010, editado por NordiCHI '10 (ACM, New York, 2010), p. 295-304.

[3] D. Cressey, Nature 544, 7648 (2017).
[4] M. A. Cavalcante, A. Bonizzia e L. C. P. Gomes, Revista Brasileira de Ensino de Física 30, 2 (2008).

[5] G. Dionisio e W. C. Magno, Revista Brasileira de Ensino de Física 29, 2 (2007).

[6] R. Hessel, C. S. de Oliveira, G. A. Santarine e D. R. Vollet, Revista Brasileira de Ensino de Física 30, 1 (2008).

[7] V. R. Coluci, G. Paulino, D. C. de Souza e E. P. R. Vasconcelos, Revista Brasileira de Ensino de Física 35, 2 (2013).

[8] F. F. Luiz, L. E. S. Souza e P. H. Domingues, Revista Brasileira de Ensino de Física 38, 2 (2016).

[9] J. T. Guaitolini Junior, G. S. Ramos, S. L. da Silva e A. C. Gama, Caderno Brasileiro de Ensino de Física 33, 2 (2016).

[10] Z. Gingl, R. Mingesz, P. Makra e J. Mellár, European Journal of Physics 32, 4 (2011).

[11] F. Laudares, M. C. S. M. Lopes e F. A. O. Cruz, Revista Brasileira de Ensino de Física 26, 3 (2004).

[12] S. T. Gobara e E. W. F. M. da Silva, Caderno Brasileiro de Ensino de Física 26 (2009).

[13] C. E. Aguiar e M. M. Pereira, Revista Brasileira de Ensino de Física 34, 3 (2012).

[14] D. A. Baldo, J. L. A. Almeida, J. M. Oliveira Jr, N. Aranha e W. Bonventi Jr, Caderno Brasileiro de Ensino de Física 33, 3 (2016).

[15] H. Cordova e A. C. Tort, Revista Brasileira de Ensino de Física 38, 2 (2016).

[16] EasyEDA - PCB design \& simulação de circuitos online - circuitos photogate, disponível em https://easyeda. com/jocoteles, acessado em 16/05/2017.

[17] GitHub - hospedagem de código para controle de versão e colaboração - códigos photogate, disponível em https: //github.com/jocoteles, acessado em 16/05/2017.

[18] Arduino - Home, disponível em https://www .arduino cc/, acessado em 04/05/2017.

[19] L. R. M. de Carvalho e H. S. de Amorim, Revista Brasileira de Ensino de Física 36, 3 (2014).

[20] A. A. M. Santos, H. S. Amorim e C. P. Dereczynski, Revista Brasileira de Ensino de Física 39, 1 (2017).

[21] S. M. Rezende, Materias e dispositivos eletrônicos (Editora Livraria da Física, São Paulo, 2004), ed. 2.

[22] Comparison of EDA software, disponível em https://en. wikipedia.org/wiki/Comparison_of_EDA_software, acessado em 04/05/2017.

[23] Welcome to Python, disponível em https://www . python. org/ acessado em 04/05/2017.

[24] What is PyQt?, disponível em https:// riverbankcomputing.com/software/pyqt/intro, acessado em 04/05/2017.

[25] Qt Designer Manual, disponível em http://doc. qt.io/qt-5/qtdesigner-manual.html, acessado em $04 / 05 / 2017$.

[26] pySerial's documentation, disponível em https: //pythonhosted.org/pyserial/, acessado em 04/05/2017.

[27] PyQtGraph - Scientific Graphics and GUI Library for Python, disponível em http://www.pyqtgraph.org/ acessado em 04/05/2017.

[28] W. Lopes, Caderno Brasileiro de Ensino de Física 25, 3 (2008).

[29] L. R. Bacon, Journal of the Franklin Institute 221, 2 (1936). 
[30] Calculate density and viscosity of glycerol/water mixtures, disponível em http://www.met.reading. ac.uk/ sws04cdw/viscosity_calc.html, acessado em $04 / 05 / 2017$.

[31] C. Sandoval, J. Caram e J. Salinas, Revista Brasileira de Ensino de Física 31, 4 (2009).

[32] M. A. Cavalcante e C. R. C. Tavoloro, Revista Brasileira de Ensino de Física 22, 2 (2000). 